\title{
Genetic optimized BP network method for camera calibration in binocular vision
}

\author{
Xingchen Hu, Honglei An, Hongxu Ma, Haibin Xie, Hongtao Xue \\ Mechatronics and Automation School \\ National University of Defense Technology \\ Changsha, 410073, China \\ E-mail: ownseven@hotmail.com
}

\begin{abstract}
Camera calibration is the first step of positioning using binocular vision. Owning to the approximation capability of the neural network, a complex mathematical model needed by traditional calibration methods can be avoided. However the general neural network methods have their drawbacks to reduce its accuracy. This paper presents searching algorithm for the best structure and parameters of a neural network using an improved genetic algorithm (GA). The experiments show that this method can be used to establish a mapping between $2 \mathrm{D}$ coordinates and 3D coordinates directly and accurately, which is better than traditional calibration and general BP network methods.
\end{abstract}

Keywords- genetic algorithms; neural network; binocular vision; $U A V$

\section{INTRODUCTION}

Binocular vision is a popular positioning configuration, and is widely used in stereo matching and 3D reconstruction. In this paper, an external binocular vision system is utilized for locating an indoor four-rotor aircraft (UAV) or even measures its attitude, and then according to the measured information, autonomous hovering control of the aircraft can be achieved. So it has higher requirements of real-time and accuracy.

Camera calibration is the first step in utilizing binocular vision for obtaining information of UAV position. In camera calibration process, it has to be identified that the optical geometry parameters of one camera interior and relative position of two cameras ${ }^{[1]}$. Because of lens distortion, installation errors and some other reasons, it is needed nonlinear modeling and solving nonlinear equations in traditional camera calibration method ${ }^{[2]}$. As a result, it can hardly satisfy the requirement of limited control feedback time.

According to some of existing camera calibration in artificial neural network (ANN) ways ${ }^{[3]}{ }^{[4]}$, especially in BP neural network methods ${ }^{[5][6]}$, it is no more needed to build any complex math model, but just can achieve a translation relationship by ANN self-learning. Once a translation method which describes coordinates from 2D to $3 \mathrm{D}$ is obtained through ANN training, it can quickly estimate the position coordinates and be suitable for control feedback. But there are some disadvantages to limit that method achieving more precise coordinates. For example, there is little theory for choosing a best network topology or initial weights and biases of ANN, so researchers hardly know which network topology and initial network weights are suitable for the problems what they are facing to, and in the training period of ANN, the tendency to be entrapped in local minimum will stop the epoch before it reaches the universal minimum point.

In the method of combining genetic algorithms (GA) with neural networks, however, the optimal network topology and initial parameters will be searched, and it can be avoided that the tendency may be entrapped in local minimum. Consequently, the efficiency and accuracy of ANN training will be enhanced. This paper, aiming at binocular vision positioning issue and the most popular ANN (BP neural network), improves encoding scheme, fitness function and genetic operators in two significant parts of ANN design and training, which are selecting network topology and optimizing weights and biases of ANN. After training, using genetic algorithms and BP neural network (GABP) method can achieve a matrix translation describing coordinates from $2 \mathrm{D}$ to $3 \mathrm{D}$ to locate the position or even attitude of UAV accurately.

In addition, this kind of method is perfectly suitable for large-scale and complex nonlinear problems to describe relationship between input and output directly.

\section{METHODS}

The main idea of this paper is obtaining the calibration relationship through off-line GA searching and BP network training, than using it to on-line position. The coordinates of each target point in two images are integrated to vectors as $\left(x_{1}, y_{1}, x_{2}, y_{2}\right)^{T}$, which are input of BP network. And the coordinates of each target point in real world as $(X, Y, Z)^{T}$ are output of BP network.

There are two main parts to use GA to optimize a BP network ${ }^{[7]}[8]$ [9]. One is network topology optimizing, which is searching for the most efficient network topology to build a BP network with the best nonlinear approach capability, and the other one is network weights and biases optimizing, which is roughly search initial values to prevent training entrapping in local minimum and enhance its self-learning capability. Some literatures have given many ideas to design GA. After many attempts, this paper designs different encoding scheme and genetic operators to fit the specialty for each part and achieves a suitable algorithm for binocular vision positioning. 


\section{A. Algorithm structure}

Firstly, it begins with network topology optimizing. In this period, a serial of chromosome is generated within some limits randomly. Secondly, it comes out various network topologies in GA ways and trains them before compare and select the best one. After choosing the best BP network, the topology structure and its connection weights are recorded and sent to the stage of network weights and biases optimizing. Thirdly, the maximum and minimum of connection weights of optimal network topology are used to build a solution space, and another serial of chromosome is generated within that space to search the best weights and biases of BP network in GA methods roughly. Finally, it gains the translation after further BP network training, which is a process that BP network tunes its parameters subtly.

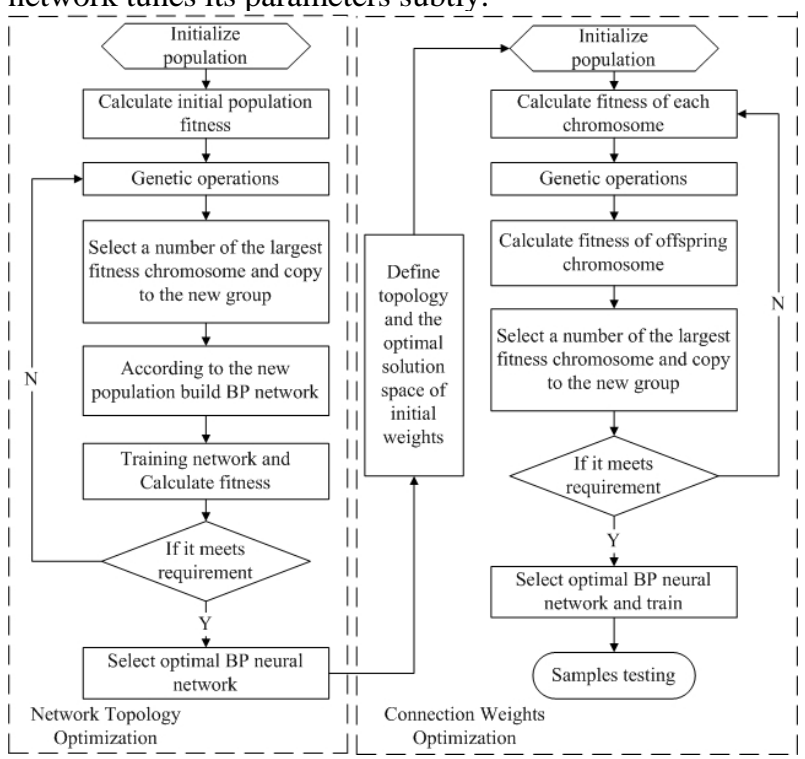

Figure 1. algorithm flow chart

\section{B. BP network topology optimizing}

The purpose of network topology optimizing is ascertaining the number of BP networks layer and neurons node numbers in each layer. According to Hornik universal approximation theorem, the standard multilayer feed-forward network with a single hidden layer, which contains finite number of hidden neurons, is a universal approximator among continuous function. But too many neurons nodes may reduce the efficiency of BP network training. Therefore, this paper searches the best network topology from 3 layers to 5 layers of BP network topology using GA.

1) Encoding scheme:

This paper uses binary coding to define the numbers of layers and nodes. Every chromosome has 20 bits. The last two positions define the layers number and the other positions separated in 3 parts define the numbers of nodes in each layer as following equation:

$$
\text { nodenum }_{j}=\sum_{i=1}^{6} 2^{i \times n_{i}}
$$

Where is the value of Position $i$ of Part $j$.

2) Fitness function:

$$
\text { fitvalue }=\left(1-\frac{\arctan (M S E / 10)}{2 \pi}\right)^{5}
$$

MSE is the error of estimation, namely the distance between coordinates by evaluated and real ones. This function helps big error receiving a small fitness value steadily and the small error awarding a large one, so the advantage of each network topology can be distinguished readily, and weeds out bad chromosome.

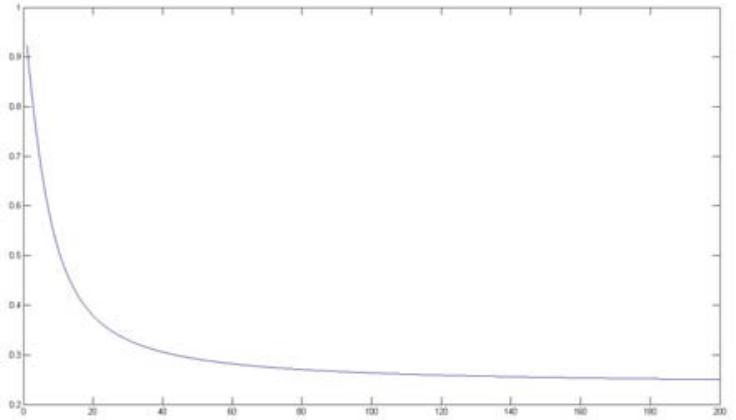

Figure 2. fitness function chart

\section{3) Selection operators:}

Roulette method is adopted, which is the most common one in GA.

\section{4) Crossover operators:}

A next generation is rebuilt by multi-point crossover to make sure its robustness.

5) Mutation operators:

Probabilistically, a position or a part of chromosome takes place $0-1$ exchange to generate a new offspring.

\section{BP network connection weight optimizing}

1) Encoding scheme:

In this stage, real number coding can boost searching ability of GA.

The length of chromosome is decided by the numbers of layers and nodes. And the value of $i^{\text {th }}$ bit calculates as follow:

$$
\text { bitvalue }_{i}=\text { rand } \times K
$$

Where rand is random digit between $[-1,1], K$ is boundary of solution space, which is settled by network topology optimizing stage.

2) Fitness function \& Selection operators:

This is the same as the network topology optimizing stage.

3) Crossover operators:

The offspring generates as follow:

$$
\text { son }=\text { father }_{1}+a \times\left(\text { father }_{2}-\text { father }_{1}\right)
$$


Where $a$ is a scaling factor, which comes out randomly between $[-d, 1+d]$. In this paper, $d$ is chosen 0.25 . It needs to give a different value to $a$ in each bit of generation.

4) Mutation operators:

The mutate offspring generates as follow:

$$
X_{n}=X_{o} \pm 0.5 \times K \times \sum_{i=0}^{m=1} \frac{a(i)}{2^{i}}
$$

Where value $a(i)$ is given 1 as 0.05 probability and given 0 as 0.95 . $K$ is the boundary of solution space. $X_{o}$ is the bit value before mutation, while $X_{n}$ is the value after that.

\section{EXPERIMENT}

\section{A. Experiment facilities and data obtaining}

The experiment of this paper uses PC with Intel Pentium 4 CPU (2.4GHz) and $1 \mathrm{G}$ internal storage. The computer runs Windows XP SP3 system and uses the library of MATLAB to train the BP network. Two cameras which are used to capture images are GC660C color digital camera produced by Prosilica Company. A point of laboratory room is set to be the original point of real world coordination. That equipment arranges as the figure 2 shows:

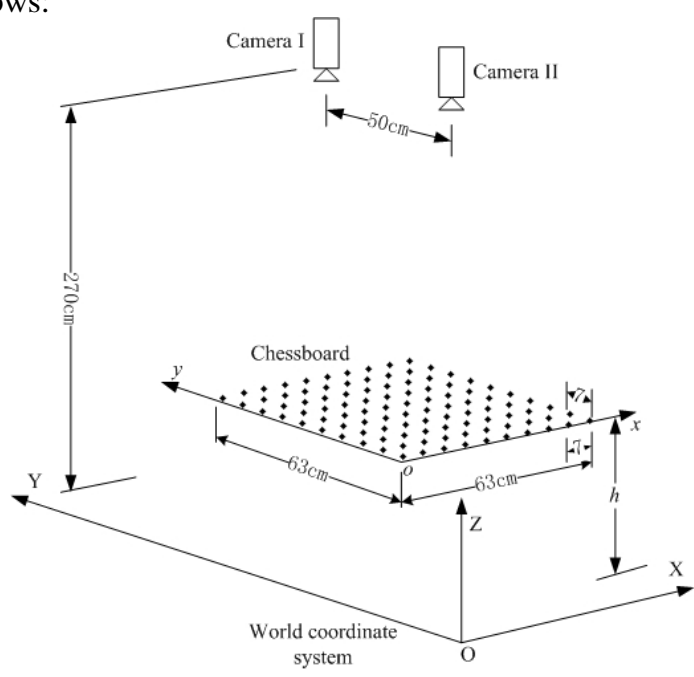

Figure 3. Equipment arrangement

The hover height of UAV is set at $70 \mathrm{~cm}$, so in this experiment, 9 groups of data at $70 \mathrm{~cm}$ height (as the $h$ shows) and 3 groups of data at $140 \mathrm{~cm}$ height are collected to train BP network. Besides, one group of data at $71 \mathrm{~cm}$ height and one group at $140 \mathrm{~cm}$ height are collected to test the trained BP network. Each group of sample point data is indicated as angular point of a chessboard in that picture. The sample collected through moving the chessboard. This experiment uses improved Harris algorithm to extract coordinates ${ }^{[10]}$.

\section{B. Results using BP network}

The experiment has tested some network topologies in literature [4] [5] [6], and the best one is literature [5], which BP network is designed as 4-9-3, namely 4 input, 3 output and 9 nodes in hidden layer. 0.670 performance of progress is taken after training the network for 1280 epochs.

\section{Result using improved GA and BP network}

TABLE I. GA PARAMETERS FOR OPTIMIZING BP NETWORK

\begin{tabular}{|c|c|c|}
\hline Parameters name & $\begin{array}{c}\text { topology } \\
\text { select }\end{array}$ & $\begin{array}{c}\text { weights } \\
\text { select }\end{array}$ \\
\hline Population size & 50 & 50 \\
\hline Chromosome length & 20 & --- \\
\hline Crossover probability & 0.6 & 0.75 \\
\hline Mutation probability & 0.01 & 0.05 \\
\hline Evolution iteration number & 100 & 100 \\
\hline
\end{tabular}

GA parameters are selected as table I shows, the best network topology of BP network is 4-4-18-3. 0.009 performance of progress is taken after training the network for only 446 epochs.

MSE of BP network method and GABP method are indicted as figure $3 \& 4$. Those points present value of each MSE and horizontal lines present value of average MSE. As these pictures showing, GABP method estimation accuracy is much better than BP network method.

Estimation results are compared further as table 2 expressed:

TABLE II. SEVERAL POSITION METHODS COMPARISON

\begin{tabular}{|c|c|c|c|}
\hline & GABP & $\boldsymbol{B P}^{[5]}$ & Method $^{[2]}$ \\
\hline Average error & $0.994 \mathrm{~cm}$ & $3.677 \mathrm{~cm}$ & $1.925 \mathrm{~cm}$ \\
\hline Error on x-axis & $0.14 \mathrm{~cm}$ & $1.65 \mathrm{~cm}$ & - \\
\hline Error on y-axis & $0.19 \mathrm{~cm}$ & $1.74 \mathrm{~cm}$ & - \\
\hline Error on z-axis & $0.98 \mathrm{~cm}$ & $2.92 \mathrm{~cm}$ & - \\
\hline Error variance & 6.659 & 25.25 & 17.82 \\
\hline Positioning time & $0.017 \mathrm{~ms}$ & $0.017 \mathrm{~ms}$ & $5 \mathrm{~ms}$ \\
\hline
\end{tabular}

It shows that speed and accuracy of binocular vision positioning are improved greatly in GABP method than $\mathrm{BP}^{[5]}$ and traditional method ${ }^{[2]}$.

The reason why the average error of the second test is bigger than the first one is that the first test is more familiar with training samples. Furthermore, the number of samples on $\mathrm{x}$-axis and y-axis are more than z-axis, so the estimation error on z-axis is bigger than others. The conclusion is the selection and quantity of samples affect the training result at a certain extent.

\section{CONCLUSION}

This paper represents a BP neural network optimized by improved genetic algorithms method is applied to binocular vision positioning. GA improves a selecting method for network topology and weights of BP networks and accelerates the convergence of neural network training. Consequently, it achieves an exact translation relationship from coordinates of $2 \mathrm{D}$ images to $3 \mathrm{D}$ world, which 
satisfies the requirements of real-time and accuracy for UAV hover control.

In addition, the selection and quantity of samples affect the training result at a certain extent. Thus, the application of this method should collect training samples containing all the concerned space to achieve a better result.

\section{REFERENCES}

[1] Tsai R Y. Versatile camera calibration technique for high accuracy 3D machine vision metrology using off-the-shelf TV cameras and lenses. IEEE Journal of Robotics and Automation, 1987, 3 (4) :3232344

[2] Shaowu Yong. Based on the binocular vision recognition and tracking of table tennis. National University of Defense Technology Master's degree thesis. 2009.11

[3] Qurban Memon, Sohaib Khan. Camera calibration and threedimensional world reconstruction of stereo-vision using neural networks. International Journal of Systems Science. Vol. 32. 11551159. 2001.

[4] Xijun Hua, Yongchao Li, Muju Wang, Lechun Xia, Yonghong Jiang. Research of camera calibration based on BP neural network. Machinery Design \& Manufacture, 2010.11. 51-53
[5] Qingjie Zhao, Zengqi Sun, Li Lan. Neural network technique in camera calibration. Control and Decision. Vol. 17 No. 3. 2002.5.

[6] Ke Zhang, Bin Xu, Lixin Tang, Hanmin Shi. BP neural network based camera calibration of binocular vision system. Mechanical and electronic. 2005. 12-15

[7] Xiaoping Wang, Liming Cao. Genetic algorithm--the theory, application and software implementation. Xi'an: Xi'an Jiao Tong University Press, 2002

[8] Frank H. F. Leung, H. K. Lam, S. H. Ling, Peter K. S. Tam. Tuning of the Structure and Parameters of Neural Network Using an Improved Genetic Algorithm. IEEE Transactions on Neural Networks, Vol. 14, 2003.1

[9] Yi Chai, Hongpeng Yin, Dajie Li, Ke Zhang. Improved genetic algorithm based on BP neural network adaptive optimal design. Journal of Chongqing University (Natural Science Edition). 2007.4. 91-96.

[10] An Cui, Zhi Yuan, Longshan Wang. Adaptive neural network based on binocular camera calibration. Computer engineering and Applications, 2009, 45(21).

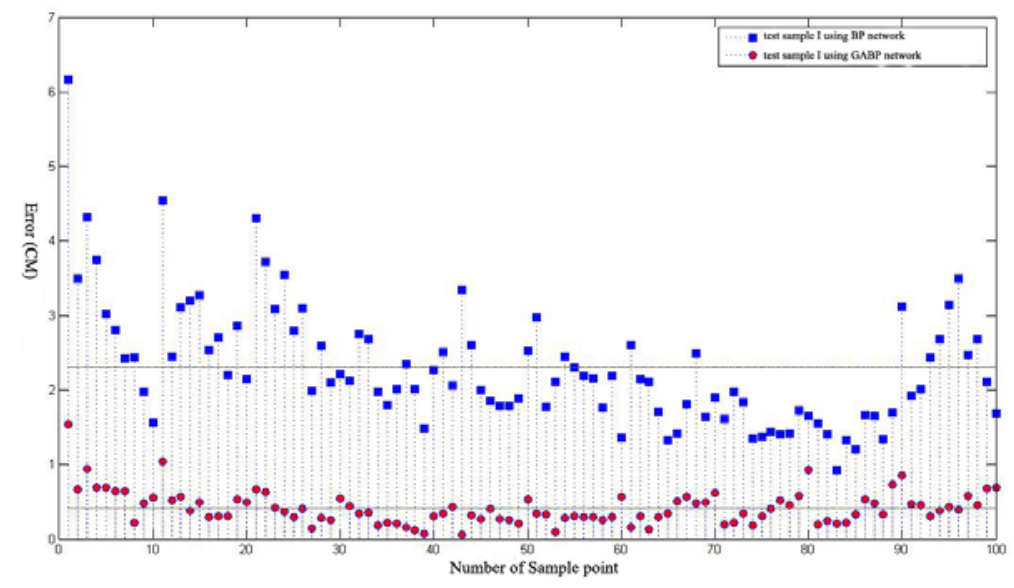

Figure 4. BP \& GABP test error of test sample I

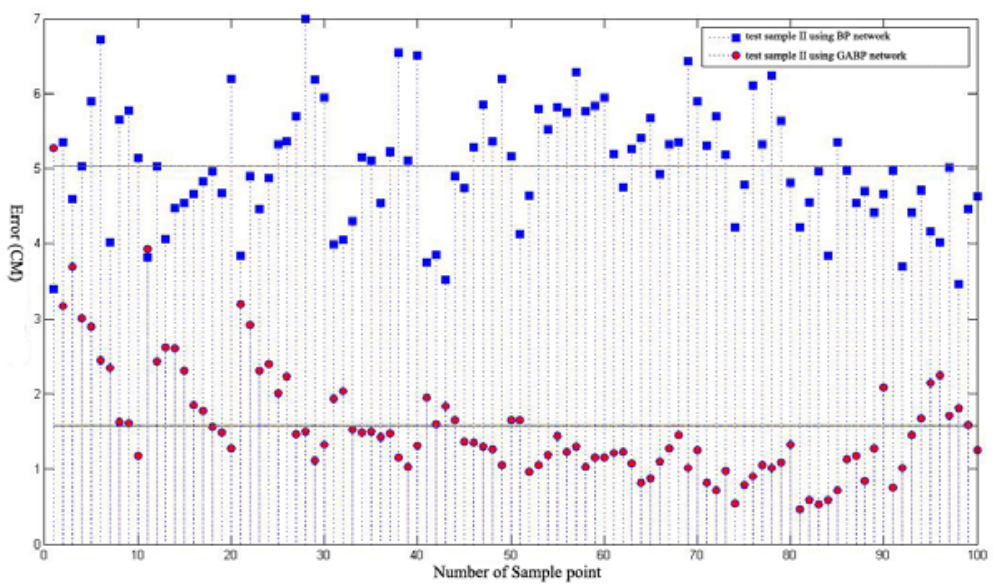

Figure 5. BP \& GABP test error of test sample II 